\title{
Novel viral vectors utilizing intron splice- switching to activate genome rescue, expression and replication in targeted cells
}

\author{
Liane Viru', Gregory Heller ${ }^{1}$, Taavi Lehto ${ }^{1}$, Kalle Pärn', Samir El Andaloussi ${ }^{1,2,3}$, Ülo Langel ${ }^{1,2}$ and Andres Merits ${ }^{1 *}$
}

\begin{abstract}
Background: The outcome of virus infection depends from the precise coordination of viral gene expression and genome replication. The ability to control and regulate these processes is therefore important for analysis of infection process. Viruses are also useful tools in bio- and gene technology; they can efficiently kill cancer cells and trigger immune responses to tumors. However, the methods for constructing tissue- or cell-type specific viruses typically suffer from low target-cell specificity and a high risk of reversion. Therefore novel and universal methods of regulation of viral infection are also important for therapeutic application of virus-based systems.

Methods: Aberrantly spliced introns were introduced into crucial gene-expression units of adenovirus vector and alphavirus DNA/RNA layered vectors and their effects on the viral gene expression, replication and/or the release of infectious genomes were studied in cell culture. Transfection of the cells with splice-switching oligonucleotides was used to correct the introduced functional defect(s).

Results: It was demonstrated that viral gene expression, replication and/or the release of infectious genomes can be blocked by the introduction of aberrantly spliced introns. The insertion of such an intron into an adenovirus vector reduced the expression of the targeted gene more than fifty-fold. A similar insertion into an alphavirus DNA/RNA layered vector had a less dramatic effect; here, only the release of the infectious transcript was suppressed but not the subsequent replication and spread of the virus. However the insertion of two aberrantly spliced introns resulted in an over one hundred-fold reduction in the infectivity of the DNA/RNA layered vector. Furthermore, in both systems the observed effects could be reverted by the delivery of splice-switching oligonucleotide(s), which corrected the splicing defects.

Conclusions: Splice-switch technology, originally developed for genetic disease therapy, can also be used to control gene expression of viral vectors. This approach represents a novel, universal and powerful method for controlling gene expression, replication, viral spread and, by extension, virus-induced cytotoxic effects and can be used both for basic studies of virus infection and in virus-based gene- and anti-cancer therapy.
\end{abstract}

\section{Background}

Viruses that infect vertebrate cells are capable of recognizing host cells and executing gene expression, genome replication and virion formation. The ability to use and control these processes is at the origin of virus-based gene-technology applications, such as the delivery of transgenes or the use of viruses for vaccinal or vaccinecarrier purposes. Based on their inherent ability to kill

\footnotetext{
* Correspondence: andres.merits@ut.ee

${ }^{1}$ Institute of Technology, University of Tartu, 50411, Tartu, Estonia Full list of author information is available at the end of the article
}

cancer cells, a number of viruses have been tested as anticancer agents, including those with double- $[1,2]$ and single-stranded DNA genomes [3] and viruses with double- [4], negative- [5] and positive-stranded [6] RNA genomes. Typically, these viruses can also infect normal cells; engineering specificity for cancer cells is therefore crucial. Several strategies have been tested for this purpose, e.g., the creation of p53-sensitive mutants [7], the alteration of viral receptor specificity $[8,9]$, the use of cancer-specific promoters in recombinant viruses $[10,11]$ and the regulation of gene expression in oncolytic viruses using cellular microRNAs $[12,13]$. The general

\section{C) Biomed Central}


shortcomings of such viral vectors are their specificity to only certain cancer types and an attenuated oncolytic potential. In addition, genetically modified viruses, especially those with RNA genomes, tend to revert, or compensate for the introduced changes, which are usually unfavorable for the infection cycle. Therefore, new approaches are needed that are applicable to different viral systems and allow for the possibility of combination with other regulation strategies without compromising the anticancer properties of the vector.

Adenoviruses (family Adenoviridae) are currently the most extensively used oncolytic DNA viruses. They have non-enveloped virions, linear double-stranded genomes and replicate in the nucleus of infected cells [14]. Adenovirus gene expression, replication, virion formation and cytotoxicity are dependent on the E1A proteins expressed via a constitutively active promoter $[15,16]$. In many engineered adenovirus vectors, including the human adenovirus 5-based Adeasy system [17], the E1A region has been deleted and, therefore, these vectors are not capable of replication unless the E1A proteins are co-expressed.

Among positive-strand RNA viruses, the alphaviruses (family Togaviridae), including Semliki Forest virus (SFV), represent one of the most promising candidates for vaccine and anticancer vectors [6]. Alphaviruses form cytoplasmic, membrane-associated replicase complexes $[18,19]$ and encode for two polyprotein precursors: a nonstructural (ns) polyprotein (replicase), translated directly from genomic RNA, and a structural polyprotein, from subgenomic (SG) mRNA [20]. The alphavirus-based replication-competent vectors contain a complete viral genome and one or more foreign-protein-encoding sequences $[6,21]$, whereas replicon vectors lack the region coding for viral structural proteins and are unable to spread [22]. Typically, the RNA genomes of alphavirus-based vectors are rescued from cloned infectious cDNA by in vitro transcription [23]. Alternatively, cDNA can be flanked with eukaryotic transcription sequences; here, rescue involves cellular transcription by RNA polymerase II and nuclear exit of the RNA product followed by translation and subsequent replication. These vectors are commonly known as DNA/RNA layered vectors [24].

With the exception of poxviruses, all DNA viruses use splicing for the expression of some of their mRNAs. Therefore, the discovery that splicing patterns can be regulated by antisense splice-switching oligonucleotides (SSOs) [25-28] created novel possibilities for regulating viral gene expression and replication. In contrast, positive-strand RNA viruses never utilize splicing; however, the insertion of introns into their cDNA sequences is often used for the construction of corresponding DNA/ RNA layered vectors [29-34]. Thus, all these viral vectors can be engineered to contain aberrantly spliced introns that block the expression of correct gene products (DNA viruses) or the rescue of infectious RNAs (DNA/RNA layered vectors). Such introns frequently occur in nature; for example, human beta thalassemia is often caused by a single-nucleotide mutation within an intronic segment of the human beta-globin gene, creating an aberrant splicing site $[35,36]$. Targeting aberrant splicing sites with antisense SSOs has resulted in efficient correction of splicing in both in vitro $[37,38]$ and in vivo models $[39,40]$.

Here, we present evidence that insertion of aberrantly spliced introns into the genome of a recombinant adenovirus vector results in severely defective marker-gene expression. Similar manipulations of DNA/RNA layered SFV vectors also resulted in a reduction of the rescue of infectious transcripts. Both of these defects were reversed by SSOs. In essence, the presence of such introns in crucial regions of the viral vector represents a lethal mutation and SSOs represent an artificial and efficient cofactor required by the constructed vector. This approach represents a novel, universal and powerful method for controlling gene expression, replication, viral spread and, by extension, virus-induced cytotoxic effects.

\section{Methods}

\section{Cells and media}

$\mathrm{HeLa}$ cells were grown at $37^{\circ} \mathrm{C}$ with $5 \% \mathrm{CO}_{2}$ in Iscove's modified Dulbecco's medium (IMDM; Invitrogen, Carlsbad, CA, USA) supplemented with $10 \%$ fetal calf serum (PAA, Pasching, Austria), $100 \mathrm{U} / \mathrm{ml}$ penicillin and 0.1 $\mathrm{mg} / \mathrm{ml}$ streptomycin. HEK293 cells were grown in the same conditions but in Dulbecco's Modified Eagle Medium (DMEM; Invitrogen). BHK-21 cells were grown in Glasgow's Minimal Essential Medium (GMEM; Invitrogen) containing $7.5 \%$ fetal calf serum, $2 \%$ tryptose phosphate broth (TPB), $200 \mathrm{mM}$ HEPES, $100 \mathrm{U} / \mathrm{ml}$ penicillin and $0.1 \mathrm{mg} / \mathrm{ml}$ streptomycin.

\section{Luciferase reporters and introns designed for insertion into DNA/RNA layered vectors}

The luciferase reporter Luc7 contains a second intron from the human beta-globin gene with a thalassemic T705G mutation (IVS2 ${ }^{705}$ ). Its sequence corresponds exactly to the reporter from the previously described pLuc/705 plasmid [38]. All other luciferase reporters were created by site-directed mutagenesis. To obtain LucWT, the T705G mutation was reverted; for Luc6+7, the mutation C654T (IVS2 ${ }^{654}$ ) [36] was added; and for Luc0, the intron was deleted.

For the insertion of the wild-type (wt) or mutant introns (with C654T, T705G or C654T + T705G mutations) into SFV DNA/RNA layered vectors, the synthetic DNA fragments running from unique XbaI $(6,637)$ to 
BglII $(6,712)$ recognition sites were obtained from GeneArt AG (Regensburg, Germany) and inserted into SFV cDNA. Intron was positioned between nucleotides 6,696 and 6,697 (all nucleotide positions are given with respect to the sequence of an infectious cDNA clone designated pSFV4 [23]).

\section{Construction, propagation and use of adenovirus vectors containing luciferase reporter}

Recombinant adenoviruses expressing firefly luciferase from reporter genes containing wt or mutant introns were constructed using the Adeasy system. The sequences of LucWT, Luc7 or Luc6+7 reporters were PCR amplified and cloned into the pShuttle-CMV vector using the restriction sites $\mathrm{KpnI}$ and NotI. Recombination with the Adeasy1 plasmid, transfection of HEK293 cells and rescue of the recombinant adenovirus was performed as previously described [17]. Adenovirus stocks were propagated by one passage in HEK293 cells; virus particles were collected, purified and titrated using an endpoint-dilution method.

For infection experiments, HeLa cells grown on a 24well plate (with a growth area of $2 \mathrm{~cm}^{2}$ per well) to $80 \%$ confluence were infected with virus stock at $0.5 \mathrm{PFU} /$ cell in $200 \mu \mathrm{l}$ serum-free IMDM media. Cells were incubated with the virus for $2 \mathrm{~h}$ at $37^{\circ} \mathrm{C}$, after which $1 \mathrm{ml}$ of complete IMDM media was added. Cells were incubated for an additional $24 \mathrm{~h}$ at $37^{\circ} \mathrm{C}$, collected and lysed, and luciferase activity was then measured using a Luciferase assay system (Promega, Madison, WI, USA).

\section{Construction of SFV DNA/RNA layered replicon and replication-competent vectors}

Recombinant SFV DNA/RNA layered-replicon and replication-competent vector plasmids were based on the previously described PCMV-SFV1 and pCMV-SFV4 [29], respectively. The luciferase gene with an intron was inserted into the region of the cDNA corresponding to the nsP3 gene of SFV [41]. In the case of vectors with two introns, the second intron was inserted into the cDNA region corresponding to the SFV nsP4 gene. If the primary transcript remains unspliced or is aberrantly spliced, these introns interrupt the reading frame encoding for viral replicase, resulting in a lethal defect. The combinations of inserted introns and the designations of the corresponding vectors are listed in Table 1.

In DNA/RNA layered replicon vectors, the sequence encoding for EGFP was placed under the control of the SFV SG promoter so its expression was strictly dependent on the ability of the vector to initiate RNA replication and transcription in transfected cells. In addition, variants from all replicon vectors were prepared by the introduction of a frame-shift (FS) mutation (4 b insertion) into the replicase region (at position 6,138) of SFV.
Table 1 Combinations of wild-type (WT) and aberrantly spliced introns used in SFV DNA/RNA layered vectors.

\begin{tabular}{rcccc}
\hline $\begin{array}{c}\text { Luc gene } \\
\text { Intron in nsP4 }\end{array}$ & Luc0 & LucWT & Luc7 & Luc6+7 \\
\hline None & $\mathrm{nc}$ & $\mathrm{A} 1$ & $\mathrm{~A} 2$ & $\mathrm{A3}$ \\
\hline $\mathrm{WT}$ & $\mathrm{B} 0$ & $\mathrm{~B} 1$ & $\mathrm{Nc}$ & $\mathrm{nc}$ \\
\hline $\mathrm{C} 654 \mathrm{~T}$ & $\mathrm{C} 0$ & $\mathrm{nc}$ & $\mathrm{C} 2$ & $\mathrm{nc}$ \\
\hline $\mathrm{T} 705 \mathrm{G}$ & $\mathrm{D} 0$ & $\mathrm{nc}$ & $\mathrm{D} 2$ & $\mathrm{nc}$ \\
\hline $\mathrm{C} 654 \mathrm{C}+\mathrm{T} 705 \mathrm{G}$ & $\mathrm{E} 0$ & $\mathrm{nc}$ & $\mathrm{Nc}$ & $\mathrm{E} 3$ \\
\hline
\end{tabular}

nc: vector not constructed.

The designs of all the DNA/RNA layered vectors are shown in Figure 1 and the sequences of all vectors and details of their construction are available from the authors on request.

\section{Antisense oligonucleotides and transfection procedures}

Phosphorothioate 2"-OMe RNA oligonucleotides designated as ON705 (5'-CCUCUUACCUCAGUUACA-3'), ON654 (5'-GCUAUUACCUUAACCCAG-3') and ONINV (5'-CCUCUUACACUCGUUACA-3') [42] were obtained from GE Healthcare (Little Chalfont, UK). If not otherwise stated, oligonucleotides were used in transfection mixtures at a final concentration of $100 \mathrm{nM}$ (in cases where two oligonucleotides were used, each were used at a final concentration of $100 \mathrm{nM}$ ) and delivered to the cells using Lipofectamine ${ }^{\mathrm{TM}} 2000$ (Invitrogen). The same reagent was also used to deliver the plasmid vectors to the cells. When cells where transfected with both oligonucleotide(s) and plasmid vectors, the oligonucleotides were always delivered $24 \mathrm{~h}$ before the transfection of the cell with plasmids.

\section{Infectious center assay (ICA) with replication-competent SFV DNA/RNA layered vectors}

ICA on the BHK-21 cells was performed as previously described $[29,43]$. A modified ICA protocol was used to estimate the infectivity of replication-competent DNA/ RNA layered vectors for HeLa cells when SFV isolates failed to form distinctive plaques. In this case, the HeLa cells were electroporated with vector DNA using a BioRad (Hercules, CA, USA) Gene Pulser II (one pulse at $220 \mathrm{~V} / 975 \mu \mathrm{F}$ ), collected and suspended in serum free growth medium. Ten-fold serial dilutions of the HeLa cell suspension were prepared in serum-free media and transferred to plates containing 90-100\% confluent BHK-21 cells. Their incubation and treatment was carried out as previously described [29].

\section{RNA purification and reveres-transcription (RT) PCR analysis}

Total RNA from transfected or infected cells was purified using TriZol reagent (Invitrogen, USA) and the 
A)

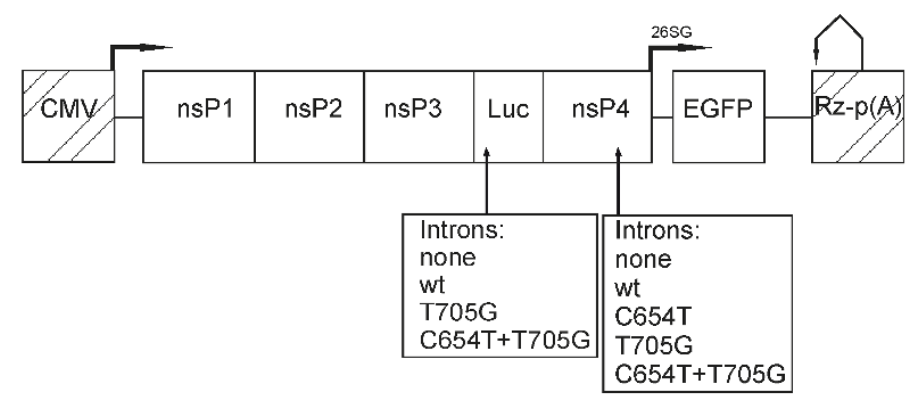

B)

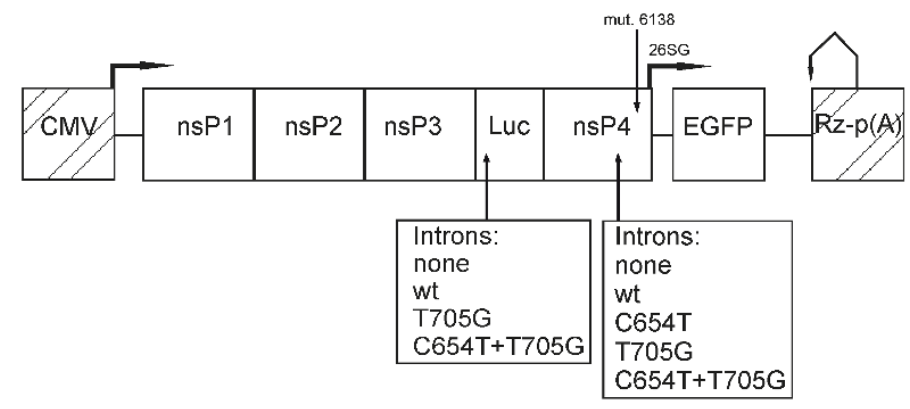

C)

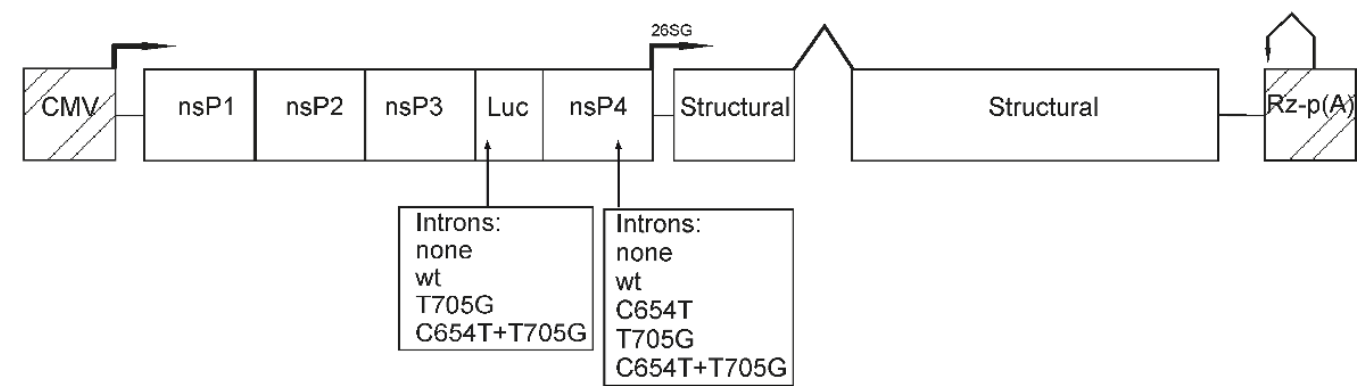

Figure 1 Schematic presentation of the SFV DNA/RNA layered vectors. The plasmid backbone of the vector is not shown; CMV immediately early promoter of human cytomegalovirus; Rz, hepatitis delta virus negative strand ribozyme; $p(A)$, simian virus 40 late polyadenylation signal. The nsP1-4, region corresponding to ns-proteins of SFV; Luc, coding sequence of the Luc reporter; 26SG, SG-promoter of SFV. Arrows in boldface indicate transcription initiation sites of the CMV and 26SG promoters; bent arrows indicate processing of mRNA transcript by ribozyme and lines indicate noncoding regions of SFV. Sites used for intron insertion are indicated by arrows; inserted introns and thalassemic mutations present in these introns are indicated under the drawings. DNA/RNA layered replicon vector without (A) or with (B) frame-shift (FS) mutation (indicated by arrow) in the region corresponding to the nsP4 gene. EGFP, coding sequence of EGFP reporter. (C) DNA/ RNA layered replication-competent vector. "Structural" indicates the region corresponding to structural genes (C-E3-E2-6K-E1) of SFV; wt intron from rabbit beta-globin gene, inserted in this region, is shown by lines above drawing.

residual DNA was removed by DNase treatment. cDNA was synthesized using First Strand cDNA synthesis kit (Fermentas; Vilnius, Lithuania), purified and used as a template for PCR amplification with primers SF (5'TGAAGAAGAGCTGTTTTTACGATCCCTTCA-3') and SR (5'-GGTTGGTACTAGCAACGCACTTTGAATTTTGTAAT-3') and Phusion Hot Start DNA polymerase (Finnzymes, Espo, Finland).

\section{Results}

Insertion of a thalassemic intron reduces gene expression from an adenovirus vector

Adenoviruses use alternative splicing to express most of their essential genes [14]. Although it is possible to replace the native intron(s) with aberrantly spliced ones, recombinant adenovirus vectors containing a Luc reporter gene with wt or aberrantly spliced introns were constructed to simplify the subsequent analysis. When the pShuttle-CMV plasmid vectors carrying LucWT or Luc705 reporters were assayed in HeLa cells, it was found that the intron with the T705G mutation reduced reporter-gene expression approximately ten-fold (data not shown). The observed reduction was nearly half that previously reported for a very similar assay system [38] and, therefore, a recombinant intron, containing the T705G and C654T mutations, was constructed and inserted into the Luc reporter. It was found that luciferase expression by Luc6+7 was approximately thirty-fold 
lower than LucWT (data not shown). This result demonstrates the strong inhibitory effect of the artificial intron, probably due to the fact that it possesses two cryptic splicing acceptor sites or because the combination of these sites activates a common cryptic splicing donor site more efficiently than a single cryptic acceptor site. In the context of the recombinant adenovirus infection, however, both aberrantly spliced introns reduced luciferase expression more than fifty-fold (data not shown), indicating that the insertion of a single aberrantly spliced intron efficiently downregulated gene expression from the viral DNA genome.

To demonstrate the effects of SSOs, HeLa cells were transfected with negative control oligonucleotide ONINV [42] or a mixture of ON705 and ON654 prior to infection with the recombinant adenovirus. Consistent with previous observations [42], it was found that ONINV slightly activated reporter expression, and we therefore excluded this from further experiments. In contrast, the mixture of SSOs acted as a much more potent activator than ONINV. The expression of the luciferase marker by AdenoLuc705 and AdenoLuc654 +705 was activated three-fold and thirteen-fold, respectively (Figure 2A).

To demonstrate that the rescue was indeed caused by splice correction, the mRNAs produced from AdenoLuc654+705 were analyzed by semiquantitative RT-PCR (Figure 2B). This assay revealed that the mRNAs from the Luc6+7 reporter were efficiently spliced because no product corresponding to unspliced RNA was detected (data not shown). In the absence of SSOs, the intron containing two thalassemic mutations was dominantly spliced using the splicing site at position 654. Only small amounts of correctly spliced products were observed and, surprisingly, no use of the incorrect splicing site at position 705 was detected (Figure 2C). Consistently, the presence of ON705 alone had only very small but detectable effect on this splicing pattern, whereas the presence of ON654 moved the splicing site to position 705 , causing a concomitant increase in the correctly spliced product (Figure 2C). Finally, the mixture of SSOs almost completely blocked the use of both incorrect splicing sites (Figure 2C). Thus, highly efficient splicing correction was likely the only mechanism behind the observed rescue of luciferase expression, which is consistent with the almost complete recovery of luciferase expression observed in the presence of SSOs (Figure 2A). Consequently, it seems reasonable to assume that gene expression from almost any DNA virus or virus-based vector can be regulated by the insertion of one or more aberrantly spliced introns in concert with splicing correction by their matching SSOs.
Insertion of aberrantly spliced introns reduces the rescue of replicating RNA from DNA/RNA layered SFV replicon vectors

The sequences of all positive-strand RNA viruses contain numerous motifs corresponding to cryptic splicing sites. In the corresponding DNA/RNA layered expression systems, these cryptic introns have a negative effect on the efficiency of the vector. Such problems could likely be solved by the insertion of efficiently spliced introns into these vectors and/or the removal of cryptic splicing sites by silent mutagenesis [29,31-34]. However, even when introns can be used in DNA/RNA layered vectors, only the rescue of replicating RNA is affected by their presence but not its subsequent replication and transcription.

To demonstrate the effect of an aberrantly spliced intron on the rescue of alphavirus replicons from DNA/ RNA layered vectors, we used SFV vectors carrying an EGFP gene under the control of the SG promoter (Figure 1A). In this system, if the introns in LucWT, Luc7 or Luc6+7 reporters are not correctly removed by splicing, the reading frame of the SFV replicase is disrupted. While the luciferase marker can be expressed from correctly spliced transcripts produced by the transcription of the vector plasmid in the cell nucleus and from replicating replicon RNAs produced in the cytoplasm, EGFP expression is here exclusively mediated by viral replicase activity (Figure 3A). Thus, the efficiency of replication can be analyzed by monitoring the expression of these markers. The corresponding assay revealed that the presence of an intron with a T705G mutation in an A2 vector (Table 1) resulted in less than a $50 \%$ reduction of luciferase expression compared to the A1 vector, whereas intron with two mutations in an A3 vector resulted in a nearly four-fold decrease of luciferase expression (Figure 3B). The much smaller effect compared to that observed for adenovirus vectors may be caused by the replicative nature of the alphavirus-replicon RNA. Consistent with this concept, it was observed that the EGFP signal appeared, albeit at later time points, in cells transfected with A2 or A3 vectors (data not shown), indicating that the initiation of RNA replication was not blocked, only delayed. To prove that differences in RNA replication were indeed responsible for the diminished difference between the vectors with wt or aberrantly spliced introns, vectors with an FS mutation, preventing expression of functional polymerase and thus any replication/transcription of replicon RNA, were constructed and analyzed. The FS mutation reduced luciferase expression approximately ten-fold (A1FS compared to A1) and, as expected, completely abolished any EGFP expression. A comparison of luciferase expression by these vectors revealed that the intron with the 


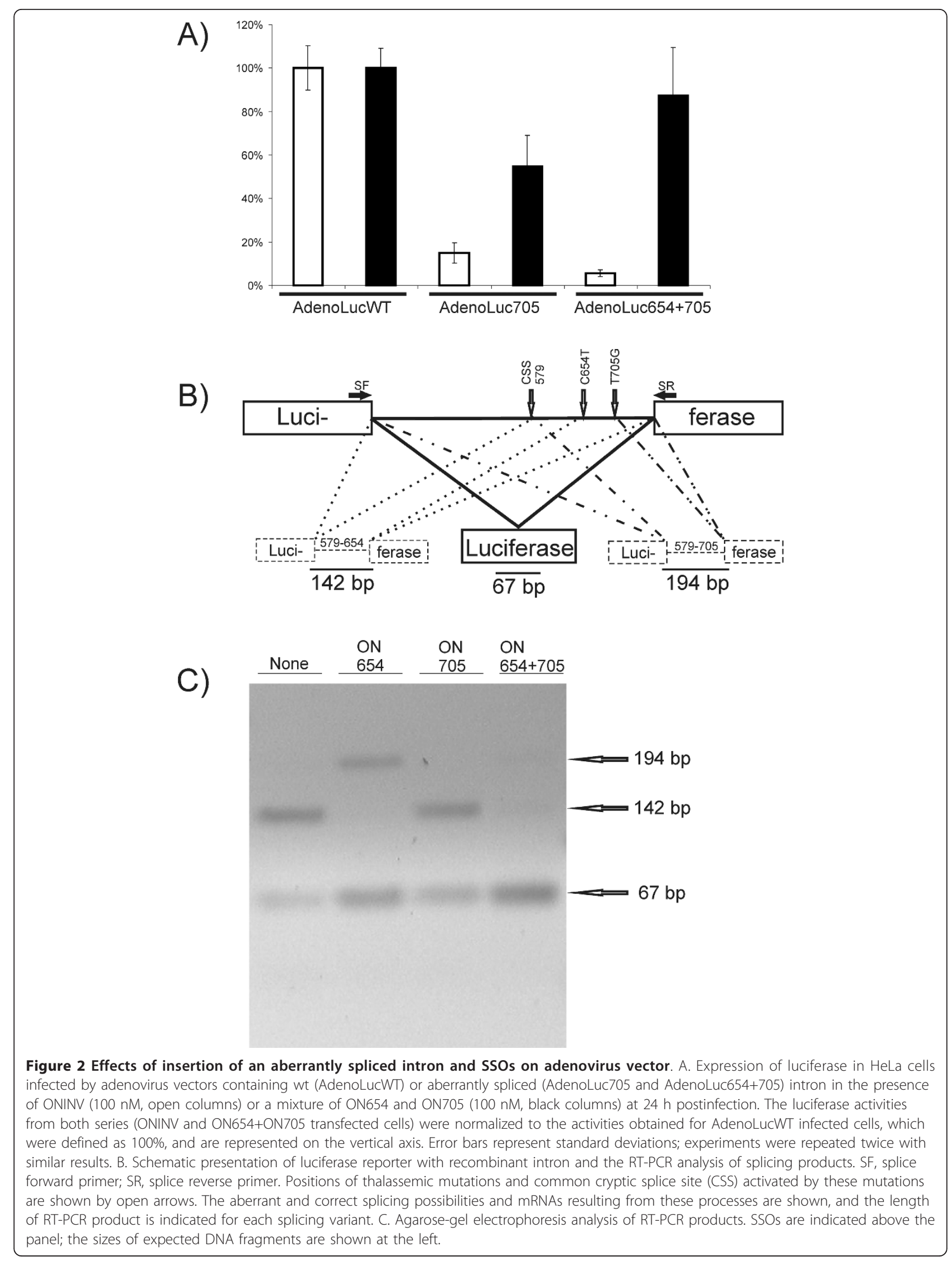


A)
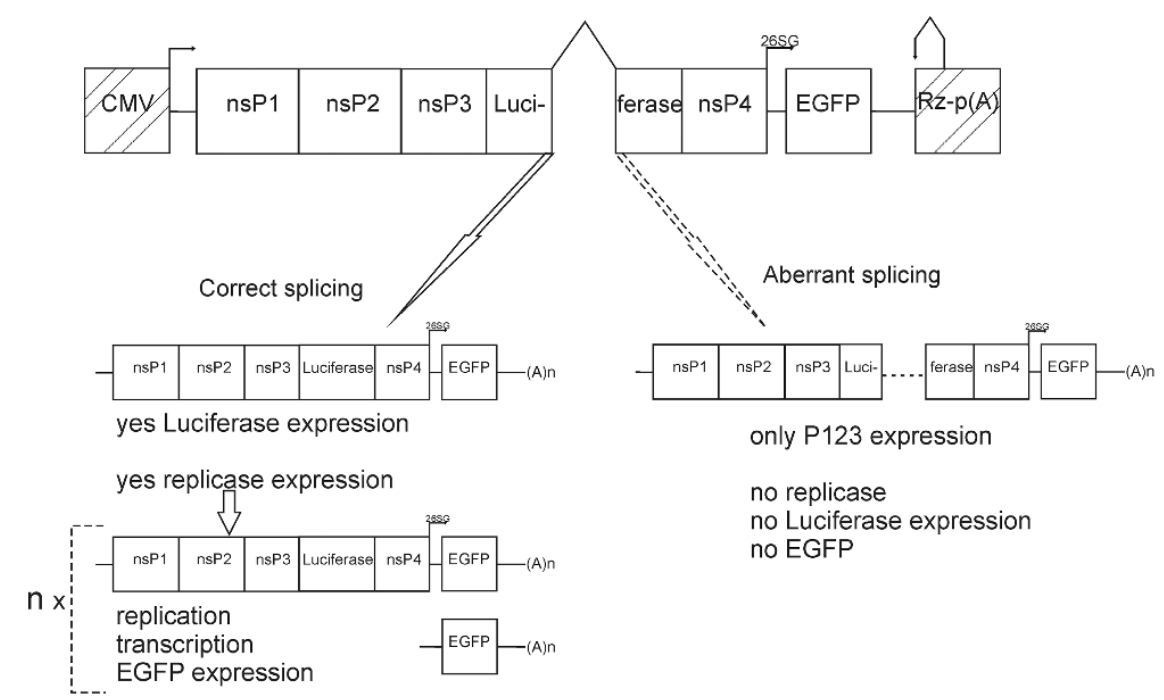

no replicase

no Luciferase expression

no EGFP

B)

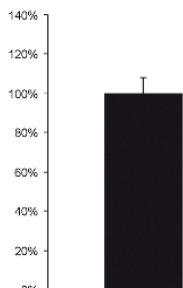

A1

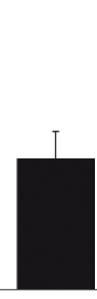

A2
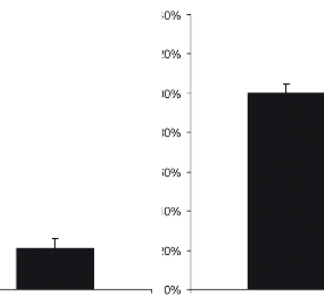

A3

A1 FS

A2 FS

A3 FS

C)

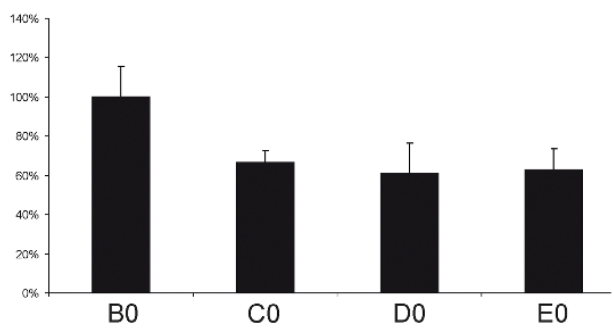

D)

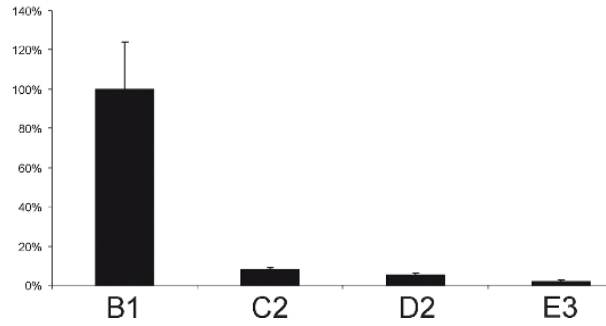

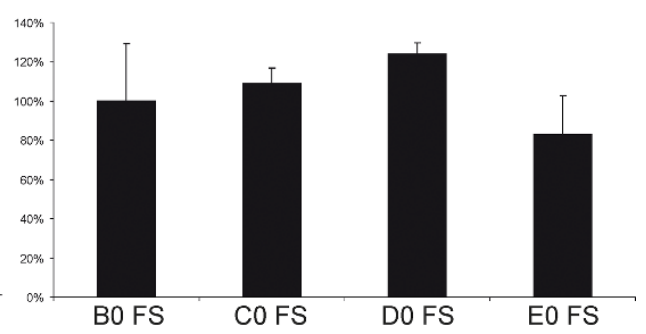

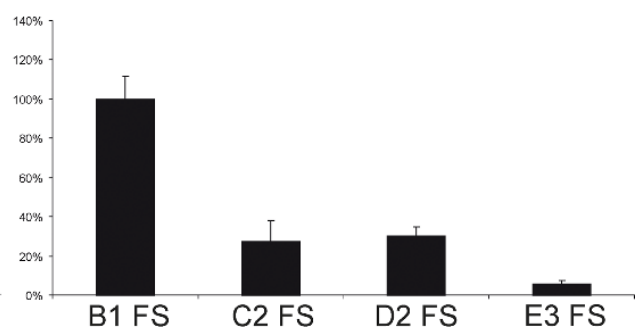

Figure 3 Effects of insertion of aberrantly spliced introns into SFV DNA/RNA layered replicon vectors. A. Schematic presentation of vectors A2 and A3 (Table 1). Products of correct splicing (left) represent mRNAs for functional replicase of SFV and for the luciferase marker; the subsequent replication of these RNAs by SFV replicase results in template amplification and transcription of SG-mRNA used for EGFP expression. mRNAs resulting from aberrant splicing (right) express only P123 polyprotein. B. Luciferase activities in HeLa cells at $24 \mathrm{~h}$ after transfection with vectors $A 1, A 2$ and $A 3$ are represented on the vertical axis; luciferase activities in cells transfected with $A 1$ vectors were taken as $100 \%$. Left panel, results for DNA/RNA layered replicon vectors; right panel, results for DNA/RNA layered replicon vectors containing FS mutation. C. Luciferase activities in HeLa cells at $24 \mathrm{~h}$ after transfection with vectors BO, CO, DO and E0 are represented on the vertical axis; luciferase activities in cells transfected with B0 vectors were taken as 100\%. Left panel, results for DNA/RNA layered replicon vectors; right panel, results for DNA/ RNA layered replicon vectors containing FS mutation. D. Luciferase activities in HeLa cells at $24 \mathrm{~h}$ after transfection with vectors B1, C2, D2 and E3 are represented on the vertical axis; luciferase activities in cells transfected with B1 vectors were taken as 100\%. Left panel, results for DNA/ RNA layered vectors; right panel, results for DNA/RNA layered vectors containing FS mutation. Error bars on panels B-D represent standard deviations; experiments were repeated three times with similar results. 
T705G mutation reduced marker expression approximately two-fold and the intron with two mutations reduced marker expression approximately eight-fold (Figure 3B). This indicates that replication is important, but not the sole factor responsible for the relatively small differences in luciferase expression between A1 and the A2 or A3 vectors. Other factors such as the reduced stability of luciferase when expressed in the SFV vector system [44] and/or the toxic effect of nsP2 of SFV on the host cells [45] may also have contributed to this effect.

Next, the effects of an intron introduced into the nsP4 encoding region of the sequence were analyzed. In this case, aberrant splicing was not expected to disrupt luciferase expression from mRNA transcripts made in the nucleus but should still abolish replicase production and, therefore, also the replication and transcription of rescued RNA. It was found that a mutant intron of any kind only caused a minor (approximately $30 \%$ ) reduction in luciferase expression (Figure 3C). This small effect most probably relates aberrant splicing did not disrupt the luciferase expression and all mRNAs, either made in nucleus or produced by viral replicase complexes, contributed to luciferase expression. Similar to the vectors $\mathrm{A} 2$ and $\mathrm{A} 3$ the EGFP expression by $\mathrm{C} 0$, D0 and E0 vectors was not abolished but slightly delayed. The experiment with corresponding vectors containing FS mutations revealed nearly twenty fold reduction of luciferase expression (BOFS compared to B0) but expectedly failed to reveal any significant effect of the intron in nsP4 region on expression of luciferase (Figure 3C).

Because insertion of one aberrantly spliced intron was clearly insufficient to suppress the release of autonomously replicating transcripts, SFV DNA/RNA layered replicon vectors containing two introns were constructed and analyzed. The results revealed that two introns with T705G mutations or a combination of introns with T705G and C654T mutations resulted in a more than ten-fold reduction of luciferase expression; two introns containing combination of C654T and T705G mutations caused a nearly one-hundred-fold reduction of marker expression (Figure 3D). Thus, combining defective introns resulted in increased inhibition of the rescue of infectious transcripts. The observed inhibitory effects were most significant early after transfection and gradually decreased over time. Coherently, the number of EGFP-positive cells, which was greatly (approximately twenty fold) reduced at the beginning of experiment, gradually increased at the later timepoints. Consistent with the data from previous experiments, it was found that in the context of DNA/RNA layered vectors with an FS mutation, the combination of these introns resulted in inhibitory effects similar to those observed for FS vectors containing a single aberrantly spliced intron in the luciferase-reporter region (compare the right panels of Figure 3B and Figure 3D).

\section{Insertion of aberrantly spliced introns reduces infectivity} of SFV DNA/RNA layered replication-competent vectors

DNA/RNA layered replicon vectors do not produce infectious progeny, and qualitative infectivity assays are thus inapplicable for these vectors. Although the inability to spread greatly increases the safety of these vectors, it also limits their use in many important applications [6]. Therefore, the effects of the insertion of aberrantly spliced introns on the infectivity of DNA/RNA layered replication-competent SFV vectors were analyzed. The vectors used in this study were based on PCMV-SFV4, which contains a wt intron from the rabbit beta-globin gene (Figure 1C). However, it was found that the insertion of one or two wt introns from the human beta-globin gene had no effect on the efficiency of infectious virus rescue from the corresponding constructs (data not shown), indicating that the different introns did not interfere with each other.

Next, a set of DNA/RNA layered replication-competent vectors containing one or two aberrantly spliced introns (Table 1) was constructed and their infectivity was tested using ICA in BHK-21 cells. The results of this analysis (Table 2) showed that the insertion of an intron with a T705G mutation into the luciferase reporter region reduced the infectivity of the vector by $50 \%$, while the insertion of an intron with two mutations resulted in a nearly five-fold drop in infectivity. The insertion of two aberrantly spliced introns caused more pronounced effects, ranging from ten-fold reductions for the $\mathrm{C} 2$ and D2 vectors to a fifty-fold reduction for the E3 vector. When ICA was performed with HeLa cells, only the constructs lacking an aberrantly spliced intron produced plaques (Table 2). Taking into account the detection limit of this experiment, we concluded that the effect of an aberrantly spliced intron or introns was greater in HeLa than in BHK-21 cells. This may result from the fact that the introns with the IVS2 $2^{705}$ and

Table 2 Infectivity of SFV replication-competent vectors in BHK-21 and HeLa cells (in PFU/pmol of vector DNA).

\begin{tabular}{rcc}
\hline $\begin{array}{c}\text { ell line } \\
\text { Construct }\end{array}$ & BHK-21 & HeLa \\
\hline $\mathrm{A} 1$ & $1.3 \times 10^{6}$ & $3.6 \times 10^{4}$ \\
\hline $\mathrm{A} 2$ & $7.6 \times 10^{5}$ & $\mathrm{Nd}$ \\
\hline $\mathrm{A} 3$ & $2.8 \times 10^{5}$ & $\mathrm{Nd}$ \\
\hline $\mathrm{B} 1$ & $1.0 \times 10^{6}$ & $2.4 \times 10^{4}$ \\
\hline $\mathrm{C} 2$ & $1.1 \times 10^{5}$ & $\mathrm{Nd}$ \\
\hline $\mathrm{D} 2$ & $1.0 \times 10^{5}$ & $\mathrm{Nd}$ \\
\hline $\mathrm{E} 3$ & $2.5 \times 10^{4}$ & $\mathrm{Nd}$ \\
\hline
\end{tabular}

nd: not detected (below $100 \mathrm{PFU} / \mathrm{pmol}$ ). 
IVS2 ${ }^{654}$ mutations had a human origin and therefore the mutations had less effect in rodent cells. Alternatively, the difference may originate from the different efficiency of the alphavirus DNA/RNA layered vector in different cell lines, as previously described for DNA/RNA layered vectors derived from the Sindbis virus [46]. Nonetheless, the infectivity of the DNA/RNA layered replicationcompetent vectors in BHK-21 cells was in accord with the luciferase expression by DNA/RNA layered replicon vectors with similar designs in HeLa cells (Table 2; Figure $3 \mathrm{~B}, \mathrm{D})$. To test whether this correlation also existed for replication-competent vectors, the corresponding vectors were transfected into HeLa cells and the luciferase activity was measured $16 \mathrm{~h}$ later. The results of this analysis (Figure 4) were consistent with those obtained using ICA; however, the correlation was lost when luciferase activities were measured $30 \mathrm{~h}$ or more post-transfection (data not shown). The presence of a single aberrantly spliced intron inside the luciferase reporter region caused three- (A2) to five-fold (A3) reductions in luciferase expression, while the combination of two aberrantly spliced introns caused a six- (C2, D2 vectors) to more than one-hundred-fold (E3) effect (Figure 4). Thus, luciferase activity can also be used to accurately estimate the infectivity of the corresponding DNA/RNA layered replication-competent vectors.

\section{Rescue of the activity of DNA/RNA replicon and} replication competent vectors by SSOs

The effect caused by the insertion of aberrantly spliced introns can be reversed through the use of SSOs with plasmid vectors in transfected cells or in stable cell lines [38] and with recombinant adenovirus vectors (Figure $2 \mathrm{~A})$. The reversion depends on the concentration of

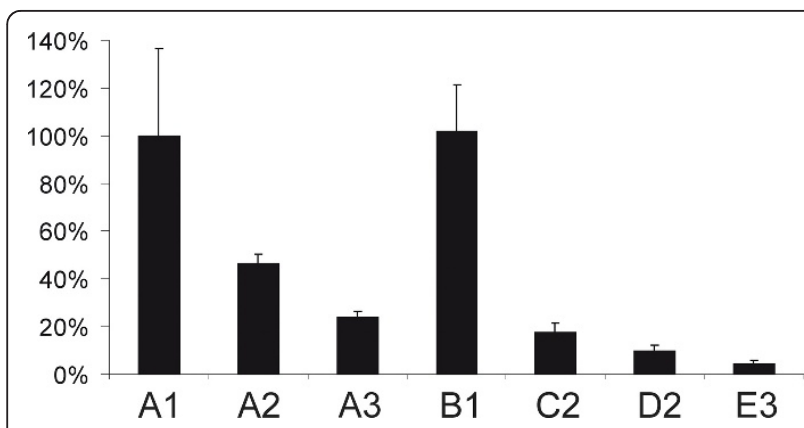

Figure 4 Effects of insertion of aberrantly spliced introns into SFV DNA/RNA layered replication-competent vectors. Luciferase activities in HeLa cells at $16 \mathrm{~h}$ after transfection with SFV replicationcompetent DNA/RNA layered vectors containing one (A1, A2 and $A 3)$ or two (B1, C2, D2 and E3) introns from the human beta-globin gene are represented on the vertical axis; luciferase activity in cells transfected with A1 vector was taken as 100\%. Error bars on represent standard deviations, the results of one reproducible experiment are shown.
SSOs [38] and can be greatly enhanced by applying oligonucleotides with splice-enhancing activities [40,47]. To determine whether this was also the case for SFV DNA/RNA layered replicon vectors, the activity of an E3 replicon was analyzed in the presence of ON705 or/ and ON654. Despite the presence of target sites for both SSOs, they were found to be unequally efficient. ON705 had, even at concentration of $100 \mathrm{nM}$, only a modest effect on the rescue of self-replicating transcripts, whereas in the presence of ON654, activity was observed rapidly, even at a concentration of $10 \mathrm{nM}$ (Figure 5). When the SSOs were combined, a synergistic effect was observed. The rescue was already efficient at $10 \mathrm{nM}$; it peaked at a $40 \mathrm{nM}$ concentration and did not change significantly at higher concentrations (Figure 5). RT-PCR analysis revealed that the splicing pattern of the intron in the E3 vector, as well as the effects of SSOs on this pattern, were identical to those observed with an adenovirus vector (data not shown). This explains, at least in part, the more prominent effect of ON654 and suggests that ON654 has some spliceenhancing activity, even though its target site does not overlap with the target of the previously characterized splice-enhancing SSO [47]. Thus, both SSOs were needed to reverse the effects caused by a combination of T705G and C654T mutations, and ON654 clearly had the greater contribution to this rescue effect.

Next, it was confirmed that SSOs were also capable of rescuing the activity of DNA/RNA layered replicationcompetent SFV vectors containing one (data not shown) or two aberrantly spliced introns (Figure 6). The presence

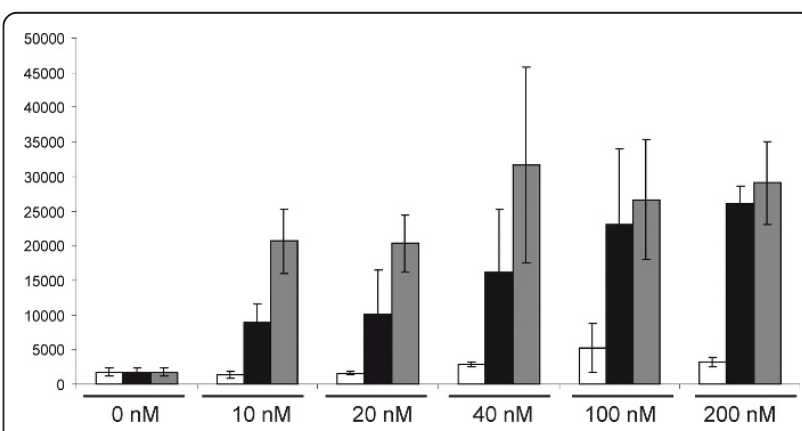

Figure 5 Rescue of the defect caused by insertion of an aberrantly spliced intron. Rescue of the defect caused by insertion of an aberrantly spliced intron into DNA/RNA layered replicon vectors by ON654, ON705 or their combination. HeLa cells were transfected with the indicated SSOs at final concentrations of $0,10,20,40,100$ or $200 \mathrm{nM} 24 \mathrm{~h}$ prior to transfection with E3 vector. Luciferase activity (in relative luciferase units) produced by the vector at $24 \mathrm{~h}$ post-transfection is represented on the vertical axis. Open columns represent activities obtained in the presence of ON705, gray columns represent activities obtained in the presence of ON654 and black columns represent activities obtained in the presence of both SSOs. Error bars represent standard deviations; the results of one of three reproducible experiments are shown. 
of these SSOs or their combination resulted in up to a two-fold activation of rescue and/or subsequent replication of the B1 vector containing only wt introns. A similar enhancement effect on SFV infection was often observed when infected cells were treated with Lipofectamine 2000 and scrambled oligonucleotides (our unpublished data). This effect may be caused by changes in the lipid composition of the plasma membranes and/or endosomal membranes used for SFV replication [19] due to lipids included in the transfection reagent. The effects of SSOs on the DNA/RNA layered replication-competent vectors containing aberrantly spliced introns were, however, much more prominent. Again, ON654 invariably mediated greater activation than did ON705 (Figure 6), even for the D2-vector bearing only T705G mutations. Consistent with prior results, the rescue was most efficient when both SSOs were applied. The magnitude of activation was over twenty times for the $\mathrm{D} 2$ and $\mathrm{C} 2$ vectors (Figure 6), clearly indicating that most of it originated from splice correction. The biggest activation (over one-hundred-fold) was observed for the E3 vector, where its activity, which in the absence of SSOs was almost at a background level, was restored to a level comparable to that of the B1 vector. These results imply that mutants with even greater defects, for example a larger number of defective introns, can probably be efficiently rescued with an appropriate mixture of SSOs.

\section{Discussion}

The basics of splice-switch technology were developed nearly two decades ago and since then the technology and its variants, such as exon skipping, have been envisioned as potent approaches to the treatment of several genetic disorders [25,48], inflammation [27] and cancer [26]. The great potential of this technology, especially evident after the first successes in in vivo applications, naturally raised the question of whether the spliceswitch platform is also applicable for viruses and virusbased vectors. One possible application would be to use it against viruses that use splicing. In this case, the SSOs can act as antiviral agents by causing deregulation of viral gene expression and subsequently block viral infection. This method may have potential as a new therapeutic approach against viruses that are difficult to target by conventional means, for example, those using latent infection accompanied with complicated splicing and minimal protein expression. However, in general, this method would be unlikely to have advantages over the inhibition of viral gene expression and replication using conventional antisense oligonucleotides or siRNAs

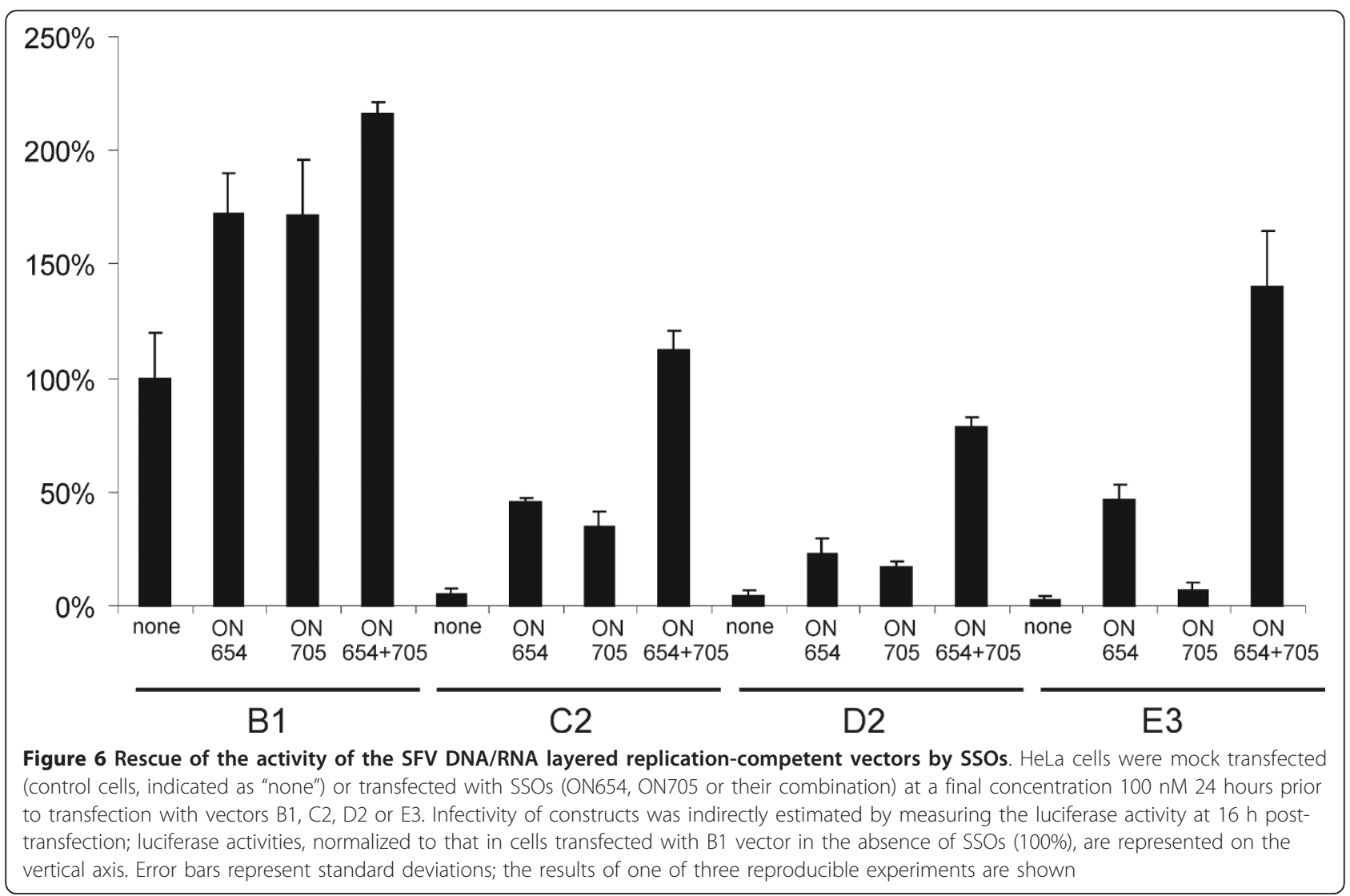


that target viral RNAs for degradation or block the expression of viral proteins [49].

An alternative possibility is to use splice-switch technology to control the release, gene expression, replication and virus-induced pathologies for genetically modified viruses, as demonstrated in this study for the adenovirus vector and layered DNA/RNA SFV vectors. Due to the different nature of the systems used, the impact of splice-switching would be different in each. When applied to an adenovirus, splice switching generates permanent control over viral infection, while in the case of DNA/RNA layered vectors, only the release of self-replicating RNA genomes or replicons can be controlled. Indeed, it was found that the introduction of aberrantly spliced introns into a gene-expression unit of a recombinant adenovirus had a stronger effect (Figure 2A) than a similar insertion made into alphavirus layered DNA/RNA vectors (Figure 3B, C, 4). The observed effect was, especially at later time points, completely lost for replication-competent SFV due to the subsequent round of infection (data not shown). Thus, the insertion of a single aberrantly spliced intron was insufficient to suppress the release of infectious replicon RNAs or viral genomes from DNA/RNA layered vectors. Therefore, it is important that the insertion of two aberrantly spliced introns into DNA/RNA layered vectors of SFV resulted in increased effect and efficiently suppressed the release of infectious RNA (Figure 3D, 4).

The infection of cells by an alphavirus can be initiated by single copy of correctly delivered and released genomic RNA. When the in vitro transcribed RNA is delivered by transfection, the efficiency is much lower (typically $\sim 10^{7} \mathrm{pfu} / \mathrm{pmol}$ of RNA), possibly reflecting the fact that transfection is not nearly as effective as natural RNA delivery in infection. That efficiency is often further reduced by approximately one log for DNA/ RNA layered vectors, probably because of the necessity to transport the DNA into the nucleus of the cell and to export produced RNA from the nucleus to the cytoplasm where it can initiate replication. Indeed, it has been demonstrated by the use of stable cell lines containing integrated copies of DNA/RNA layered vectors that the nuclear export and stability of produced RNA are likely the critical steps limiting the efficiency of DNA/RNA layered vectors [46]. It has also been suggested that RNAs made in the nucleus are intrinsically less efficient in replication initiation than those delivered by a natural infection route: single-copy transcript made in the nucleus is generally insufficient to initiate replication, instead the presence of a minimal threshold level of RNA transcripts is required [46]. RT-PCR analysis of splicing products revealed that even in the case of the "strongest" aberrantly spliced intron, a considerable amount of correctly spliced products were present, corresponding to the potentially self-replicating replicon RNAs. Additionally, the appearance of EGFP in cells transfected with such DNA/RNA layered replicon vectors was not blocked but delayed. Both of these facts are consistent with the model suggested by Boorsma et al., 2003 [46]. Additionally, the threshold level itself may be higher for DNA/RNA layered vectors containing aberrant intron(s). Data obtained for vectors with FS mutations indicates that aberrant splicing did not negatively affect the transport of mRNA from the nucleus or its stability (Figure 3C). Thus, these mRNAs, which were not capable of producing a functional replicase (Figure $3 \mathrm{~A})$ but did contain the correct cis-elements required for replication and transcription, accumulated in transfected cells. It is also known that the alphavirus replicase complex, when provided in trans, is capable of replicating such templates $[18,22]$ and, therefore, these RNA molecules may act as defective interfering genomes. The nsP1-3 proteins of SFV and their precursors are also translated from aberrantly-spliced RNAs and may have effect on the the formation of functional replicase complexes. Combined with their reduced copy number, this would likely result in a situation in which the threshold levels of correctly spliced RNAs are reached at later times. Additionally, the time taken to reach required threshold levels is limited by the loss or inactivation of vector DNA or by the death or severe damage of transfected cells due to the toxic effects of nsP2 proteins [45] translated from defective RNAs (Figure $3 \mathrm{~A}$ ). Consequently, the required RNA threshold levels may not be reached at all, as was likely the case for the HeLa cells transfected with replication-competent DNA/RNA layered vectors (Table 2). Although our data indicates that the insertion of a larger number of aberrantly spliced introns will further enhance the suppression of infectious RNA release, there may be no need to block it completely. A reduction in the rate of accumulation of correctly spliced RNAs is likely sufficient to block the release of infectious viruses.

Despite the fact that inhibition of adenovirus vector gene expression by the insertion of aberrantly spliced introns was straightforward and multiple factors contributed to reduce the release of infectious alphavirus replicons or genomes, both systems could be efficiently rescued by SSOs. Interestingly, the rescue by the combination of two SSOs was always nearly complete, and, surprisingly, more significant defects caused by aberrantly spliced intron(s) led to higher rescue efficiencies (Figure 2A, C; Figure 6). Because the rescue rate for the E3 vector (Figure 5) and D2 vector (Figure 6) by ON705 alone was low, we concluded that the presence of SSOs with splicing-enhancement activity is required to achieve high-level or even complete reversion of defects caused by the insertion of aberrantly spliced introns. 


\section{Conclusions}

The results of this study show that splice-switching is feasible for the construction of conditionally activated systems of different viruses and could be applicable in vaccination or virus-based gene or anticancer therapy. The method is straightforward and can be easily combined with other techniques used for the construction of regulated viral vectors. Unlike most manipulations made at the level of the replicating genome, the inhibition of infectious genome rescue cannot be corrected or compensated by activity of non-proofreading viral RNA replicase. Thus, the splice-switch technology can be used to achieve a targeted rescue of the viral genome from the vectors in cells where SSOs are present. However, as SSOs cannot affect the subsequent spread of alphavirus, additional control measures may be required, such as the insertion of suicide genes [50]. The possibilities for practical application of this approach depend on the development and improvement of methods for tissue- and cell-type-specific delivery of SSOs, which act as essential cofactors for the release and (in the case of DNA viruses) propagation of such viral vectors.

\section{Acknowledgements}

This research was supported by grant 7407 from the Estonian Science Foundation, target financing projects SF0180087s08 and SF0180027s08 from the Estonian Government, the DoRa Program of The European Social Fund, the Archimedes Foundation and by the European Union through the European Regional Development Fund via the Center of Excellence in Chemical Biology. SELA was partially funded by the Swedish Society for Medical Research (SSMF).

\section{Author details \\ ${ }^{1}$ Institute of Technology, University of Tartu, 50411, Tartu, Estonia. ${ }^{2}$ Department of Neurochemistry, Stockholm University, SE-106-92, Stockholm, Sweden. ${ }^{3}$ Department of Laboratory Medicine, Karolinska Institutet, SE-141 86, Huddinge, Sweden.}

\section{Authors' contributions}

LV performed experiments with replication-competent virus vectors, GH performed experiments with adenovirus and SFV replicon vectors. KP and TL participated in experiments with SSOs, SELA and ÜL developed the concept and designed approaches, AM designed experiments, supervised the project and wrote the manuscript. All authors read and approved the final manuscript.

\section{Competing interests}

LV, GH, TL, SELA, UL and AM are also co-inventors in patent application P200900077 "A method and composition for creating conditional lethality in virus mutants and for eliminating the viability of a eukaryotic cell", which describes potential industrial applications of several vectors and approaches described in this manuscript; the owner of IP rights is the University of Tartu.

Received: 22 February 2011 Accepted: 19 May 2011

Published: 19 May 2011

\section{References}

1. K Toth, D Dhar, WS Wold, Oncolytic (replication competent) adenoviruses as anti-cancer agents. Expert Opin Biol Ther. 10, 353-368 (2010). doi:10.1517/14712590903559822
2. R Kanai, H Wakimoto, T Cheema, SD Rabkin, Oncolytic herpes simplex virus vectors and chemotherapy: are combinatorial strategies more effective for cancer? Future Oncol. 6, 619-634 (2010). doi:10.2217/fon.10.18

3. J Rommelaere, K Geletneky, AL Angelova, L Daeffler, C Dinsart, I Kiprianova, RJ Schlehofer, Z Raykov, Oncolytic parvoviruses as cancer therapeutics. Cytokine Growth Factor Rev. 21, 185-195 (2010). doi:10.1016/j. cytogfr.2010.02.011

4. KJ Harrington, EM Karapanagiotou, V Roulstone, KR Twigger, CL White, L Vidal, D Beirne, R Prestwich, K Newbold, M Ahmed, K Thway, CM Nutting, M Coffey, D Harris, RG Vile, HS Pandha, JS Debono, AA Melcher, Two-stage phase I dose-escalation study of intratumoral reovirus type 3 dearing and palliative radiotherapy in patients with advanced cancers. Clin Cancer Res. 16, 3067-3077 (2010). doi:10.1158/1078-0432.CCR-10-0054

5. GN Barber, Vesicular stomatitis virus as an oncloytic vector. Viral Immunol. 17, 516-527 (2004). doi:10.1089/vim.2004.17.516

6. GJ Atkins, MN Fleeton, GJ Sheahan, Therapeutic and prophylactic applications of alphavirus vectors. Expert Rev Mol Med. 11, 33 (2008)

7. C Heise, A Sampson-Johannes, A Williams, F McCormick, DD Von Hoff, DH Kirn, ONYX-015, an E1B gene-attenuated adenovirus, causes tumor-specific cytolysis and antitumoral efficacy that can be augmented by standard chemotherapeutic agents. Nat Med. 3, 639-645 (1997). doi:10.1038/nm0697639

8. M Breidenbach, TD Rein, M Wang, DM Nettelbeck, A Hemminki, I Ulasov, AR Rivera, M Everts, RD Alvarez, JT Douglas, DT Curiel, Genetic replacement of adenovirus shaft fiber reduces liver tropism in ovarian cancer gene therapy. Hum Gene Ther. 15, 509-518 (2004). doi:10.1089/ 10430340460745829

9. S Myhre, P Henning, M Freidman, S Stahl, L Lindholm, MK Magnusson, Retargeted adenovirus vectors with dual specificity; binding specificities conferred by two different Affibody molecules in fiber. Gene Ther. 16 252-261 (2009). doi:10.1038/gt.2008.160

10. JP Latham, PF Searle, V Mautner, ND James, Prostate-specific antigene promoter/enhancer driven gene therapy for prostate cancer: construction and testing of tissue-specific adenovirus vector. Cancer Res. 60, 334-341 (2000)

11. ZX Wang, HB Bian, JS Yang, W De, XH Ji, Adenovirus-mediated suicide gene therapy under the control of Cox-2 promoter for colorectal cancer. Cancer Biol Ther. 8, 1480-1488 (2009). doi:10.4161/cbt.8.15.8940

12. RE Edge, TJ Falls, CW Brown, BD Lichty, H Atkins, JC Bell, A let-7 MicroRNAsensitive vesicular stomatitis virus demonstrates tumor-specific replication. Mol Ther. 16, 1437-1443 (2008). doi:10.1038/mt.2008.130

13. C Gomez-Manzano, J Fueyo, Oncolytic adenoviruses for treatment of brain tumors. Curr Opin Mol Ther. 12, 530-537 (2010)

14. AJ Berk, Adenoviridae: the viruses and their replication. in Fields Virology, vol. 2, ed. by Knipe DM, Howley PM (Lippincott Williams \& Wilkins/Wolters Kulwer, Philadelphia, 2007), pp. 2355-2394

15. N Jones, T Shenk, An adenovirus type 5 early gene function regulates expression of other early viral genes. Proc Natl Acad Sci USA. 76, 3665-3669 (1979). doi:10.1073/pnas.76.8.3665

16. $\mathrm{P}$ Hearing, $\mathrm{T}$ Shenk, The adenovirus type 5 E1A transcriptional control region contains a duplicated enhancer element. Cell. 33, 695-703 (1983). doi:10.1016/0092-8674(83)90012-0

17. TC He, S Zhou, LT da Costa, J Yu, KW Kinzler, B Vogelstein, A simplified system for generating recombinant adenoviruses. Proc Natl Acad Sci USA. 95, 2509-2514 (1998). doi:10.1073/pnas.95.5.2509

18. El Frolova, R Gorchakov, L Pereboeva, S Atasheva, I Frolov, Functional Sindbis virus replicative complexes are formed at the plasma membrane. J Virol. 84, 11679-11695 (2010). doi:10.1128/JVI.01441-10

19. P Spuul, G Balistreri, L Kääriäinen, T Ahola, Phosphatidylinositol 3-kinaseactin-, and microtubule-dependent transport of Semliki Forest Virus replication complexes from plasma membrane to modified lysosomes. J Virol. 84, 7543-7557 (2010). doi:10.1128/JVI.00477-10

20. L Kääriäinen, T Ahola, Functions of alphavirus nonstructural proteins in RNA replication. Prog Nucleic Acid Res Mo?? Biol. 71, 187-222 (2002)

21. K Rausalu, A lofik, L Ülper, L Karo-Astover, V Lulla, A Merits, Properties and use of novel replication-competent vectors based on Semliki Forest virus. Virol J. 6, 33 (2009). doi:10.1186/1743-422X-6-33

22. P Liljeström, $H$ Garoff, $A$ new generation of animal cell expression vectors based on the Semliki Forest virus replicon. Biotechnology (N Y). 9, 1356-1361 (1991). doi:10.1038/nbt1291-1356 
23. P Liljeström, S Lusa, D Huylebroeck, H Garoff, In vitro mutagenesis of a fulllength cDNA clone of Semliki Forest virus: the small 6,000-molecular-weight membrane protein modulates virus release. J Virol. 65, 4107-4113 (1991)

24. TW Dubensky Jr, DA Driver, JM Polo, BA Belli, EM Latham, CE Ibanez, S Chada, D Brumm, TA Banks, SJ Mento, DJ Jolly, SMW Chang, Sindbis virus DNA-based expression vectors: utility for in vitro and in vivo gene transfer. J Virol. 70, 508-519 (1996)

25. J Bauman, N Jearawiriyapaisarn, R Kole, Therapeutic potential of spliceswitching oligonucleotides. Oligonucleotides. 19, 1-13 (2009). doi:10.1089/ oli.2008.0161

26. JA Bauman, SD Li, A Yang, L Huang, R Kole, Anti-tumor activity of spliceswitching oligonucleotides. Nucl Acids Res. 38, 8348-8356 (2010). doi:10.1093/nar/gkq731

27. MA Graziewicz, TK Tarrant, B Buckley, J Roberts, L Fulton, H Hansen, H Ørum, R Kole, P Sazani, An endogenous TNF-alpha antagonist induced by splice-switching oligonucleotides reduces inflammation in hepatitis and arthritis mouse models. Mol Ther. 16, 1316-1322 (2008). doi:10.1038/ mt.2008.85

28. GJ van Ommen, J van Deutekom, A Aartsma-Rus, The therapeutic potential of antisense-mediated exon skipping. Curr Opin Mol Ther. 10, 140-149 (2008)

29. L Ülper, I Sarand, K Rausalu, A Merits, Construction, properties, and potential application of infectious plasmids containing Semliki Forest virus full-length CDNA with an inserted intron. J Virol Methods. 148, 265-270 (2008). doi:10.1016/j.jviromet.2007.10.007

30. IE Johansen, Intron insertion facilitates amplification of clones virus CDNA in Escherichia coli while biological activity is re-established after transcription in vivo. Proc Natl Acad Sci USA. 93, 12400-12405 (1996). doi:10.1073/ pnas.93.22.12400

31. SJ Yang, F Revers, S Souche, H Lot, O Le Gall, T Candresse, J Dunez, Construction of full-length cDNA clones of lettuce mosaic virus (LMV) and the effects of intron-insertions on their viability in Escherichia coli and their infectivity to plants. Arch Virol. 143, 2443-2451 (1998). doi:10.1007/ s007050050474

32. JJ Lopez-Moya, JA Garcia, Construction of a stable and highly infectious intron-containing CDNA clone of plum pox potyvirus and its use to infect plants by particle bombardment. Virus Res. 68, 99-107 (2000). doi:10.1016/ S0168-1702(00)00161-1

33. S Marillonnet, C Thoeringer, R Kandzia, V Klimyuk, Y Gleba, Systemic Agrobacterium tumifaciens-mediated transfection of viral replicons for efficient transient expression in plants. Nature Biotechnol. 23, 718-723 (2005). doi:10.1038/nbt1094

34. JM Gonzalez, Z Penzes, F Almazan, E Calvo, L Enjuanes, Stabilization of a full-length infectious cDNA clone of transmissible gastroenterits coronavirus by insertion an intron. J Virol. 76, 4644-4661 (2002)

35. M Busslinger, N Moschonas, RA Flavell, Beta+thalassemia: aberrant splicing results from a single point mutation in an intron. Cell. 27, 289-298 (1981). doi:10.1016/0092-8674(81)90412-8

36. T Jo, S Momita, N Sadamori, M Tomonaga, S Fucharoem, Y Fukumaki, M Ichimaru, A case of theta-thalassemia with a $\mathrm{C}$ - $-\mathrm{T}$ substitution at position 654 of the second intervening sequence of the beta-globin gene. Intern Med. 31, 269-272 (1992). doi:10.2169/internalmedicine.31.269

37. Z Dominski, R Kole, Restoration of correct splicing in thalassemic pre-mRNA by antisense oligonucleotides. Proc Natl Acad Sci USA. 90, 8673-8677 (1993). doi:10.1073/pnas.90.18.8673

38. SH Kang, MJ Cho, R Kole, Up-regulation of luciferase gene expression with antisense oligonucleotides: implications and applications in functional assay development. Biochemistry. 37, 6235-6239 (1998). doi:10.1021/bi980300h

39. J Roberts, E Palma, P Sazani, H Ørum, M Cho, R Kole, Efficient and persistent splice switching by systemically delivered LNA oligonucleotides in mice. Mol Ther. 14, 471-475 (2006). doi:10.1016/j.ymthe.2006.05.017

40. S Svasti, T Suwanmanee, S Fucharoen, HM Moulton, MH Nelson, N Maeda, O Smithies, R Kole, RNA repair restores hemoglobin expression in IVS2-641 thalassemic mice. Proc Natl Acad Sci USA. 106, 1205-1210 (2009). doi:10.1073/pnas.0812436106

41. N Tamberg, V Lulla, R Fragkoudis, A Lulla, JK Fazakerley, A Merits, Insertion of EGFP into the replicase gene of Semliki Forest virus results in a novel, genetically stable marker virus. J Gen Virol. 88, 1225-1230 (2007). doi:10.1099/vir.0.82436-0

42. P Guterstam, M Lindgren, H Johansson, U Tedebark, J Wangel, S El Andaloussi, ?Ü? Langel, Splice-switching efficiency and specificity for oligonucleotides with locked nucleic acid monomers. Biochem J. 412, 307-313 (2008). doi:10.1042/BJ20080013

43. R Gorchakov, E Frolova, BR Williams, CM Rice, I Frolov, PKR-dependent and -independent mechanisms are involved in translational shutoff during Sindbis virus infection. J Virol. 78, 8455-8467 (2004). doi:10.1128/ JVI.78.16.8455-8467.2004

44. M Varjak, E Žusinaite, A Merits, Novel functions of the alphavirus nonstructural protein nsP3 C-terminal region. J Virol. 84, 2352-2364 (2010). doi:10.1128/JVI.01540-09

45. N Garmashova, R Gorchakov, E Frolova, I Frolov, Sindbis virus nonstructural protein nsP2 is cytotoxic and inhibits cellular transcription. J Virol. 80, 5686-5696 (2006). doi:10.1128/JVI.02739-05

46. M Boorsma, P Saudan, H Pfruender, JE Bailey, S Schlesinger, WA Renner, FM Bachmann, Alphavirus cDNA-based expression vectors: effects of RNA transcription and nuclear export. Biotechnol Bioeng. 81, 553-562 (2003). doi:10.1002/bit.10496

47. S Resina, R Kole, A Travo, B Lebleu, AR Thierry, Switching on transgene expression by correcting aberrant splicing using multi-targeting stericblocking oligonucleotides. J Gen Med. 9, 498-510 (2007). doi:10.1002/ jgm.1044

48. QL Lu, T Yokota, S Takeda, L Garcia, F Muntoni, T Partridge, The status of exon skipping as therapeutic approach to Duchenne muscular dystrophy. Mol Ther. 19, 9-15 (2011). doi:10.1038/mt.2010.219

49. J Haasnoot, B Berkhout, Nucleic acid-based therapeutics in the battle against pathogenic viruses. Handb Exp Pharmacol. 189, 243-263 (2009). doi:10.1007/978-3-540-79086-0_9

50. JC Tseng, G Daniels, D Meruelo, Controlled propagation of replicationcompetent Sindbis viral vector using suicide gene strategy. Gene Ther. 16 291-296 (2009). doi:10.1038/gt.2008.153

doi:10.1186/1743-422X-8-243

Cite this article as: Viru et al:: Novel viral vectors utilizing intron spliceswitching to activate genome rescue, expression and replication in targeted cells. Virology Journal 2011 8:243.

\section{Submit your next manuscript to BioMed Central and take full advantage of:}

- Convenient online submission

- Thorough peer review

- No space constraints or color figure charges

- Immediate publication on acceptance

- Inclusion in PubMed, CAS, Scopus and Google Scholar

- Research which is freely available for redistribution 